Relations industrielles

Industrial Relations

\title{
Ressources humaines. Gérer les personnes et l'ordre social dans l'entreprise, Par Évelyne Léonard (2015) Louvain-La-Neuve : De Boeck Supérieur, 168 pages. ISBN : 978-2-8041-9019-4
}

\section{Sylvain Luc}

Volume 71, numéro 2, printemps 2016

URI : https://id.erudit.org/iderudit/1036617ar

DOI : https://doi.org/10.7202/1036617ar

Aller au sommaire du numéro

\section{Éditeur(s)}

Département des relations industrielles de l’Université Laval

ISSN

0034-379X (imprimé)

1703-8138 (numérique)

Découvrir la revue

Citer ce compte rendu

Luc, S. (2016). Compte rendu de [Ressources humaines. Gérer les personnes et l'ordre social dans l'entreprise, Par Évelyne Léonard (2015) Louvain-La-Neuve : De Boeck Supérieur, 168 pages. ISBN : 978-2-8041-9019-4]. Relations

industrielles / Industrial Relations, 71(2), 379-381.

https://doi.org/10.7202/1036617ar

Tous droits réservés (C Département des relations industrielles de l’Université Laval, 2016
Ce document est protégé par la loi sur le droit d'auteur. L’utilisation des services d'Érudit (y compris la reproduction) est assujettie à sa politique d'utilisation que vous pouvez consulter en ligne.

https://apropos.erudit.org/fr/usagers/politique-dutilisation/ 
des interactions entre modes de régulations aux stratégies divergentes, voire opposées, entre entreprises et travailleurs aux prises avec ces «démarches compétences».

Il s'agit donc d'un recueil d'analyses précises sur l'interaction entre acteurs dans les régulations de la relation salariale, celles-ci se déclinant à de multiples versions selon l'angle abordé. Sont, en effet, abordées tour à tour les dimensions du rôle de l'État, des syndicats, de leurs fédérations en branches (ou autres comités sectoriels selon le territoire), de l'importance du déploiement territorial, des logiques d'activation, des tensions entre agendas articulés par des dispositifs supranationaux et des contraintes issues de périodes de crises... Ce volume s'avèrera une lecture extrêmement stimulante pour des chercheurs et étudiants en relations industrielles, sociologie du travail, économie politique... Ces textes d'introduction, de table ronde d'acteurs et de conclusion aident à la synthèse et au prolongement des réflexions, riches de thématiques variées et nuancées.

\section{Jean-Luc Bédard}

Professeur

Département Éducation, TÉLUQ

\section{Ressources humaines. \\ Gérer les personnes et l'ordre social dans l'entreprise}

Par Évelyne Léonard (2015) Louvain-LaNeuve: De Boeck Supérieur, 168 pages. ISBN : 978-2-8041-9019-4.

D'entrée de jeu, Evelyne Léonard nous annonce avoir longtemps hésité à rédiger cet ouvrage, par peur qu'il ne soit relégué au statut de «livre de plus sur des tablettes parfois bien encombrées». Cette crainte est légitime: depuis plusieurs années, voire plusieurs décennies, les ouvrages de référence destinés à l'enseignement de la gestion des ressources humaines (et de la gestion au sens large) se suivent et se ressemblent. Rapidement, nous pouvons les classer en trois grandes catégories. Les premiers, orientés autour de perspectives prescriptives et normatives, suggèrent que la gestion des ressources humaines constitue un ensemble de pratiques universelles qui peuvent se décliner en tout temps et en tout lieu, nonobstant les contextes organisationnels, institutionnels et culturels. Les deuxièmes, valorisant une certaine conception stratégique de la $\mathrm{GRH}$, proposent que les contingences environnementales déterminent les conditions de sa mise en application; les pratiques de GRH doivent être alignées sur la stratégie de l'entreprise et cohérentes entre elles. Partant, dans des contextes similaires, nous retrouvons les mêmes pratiques; I'universalité est ici relative. Enfin, nous retrouvons dans la troisième et dernière catégorie, des ouvrages plus critiques, voire pamphlétaires, décriant ces conceptions normatives et prescriptives et soulignant, au départ d'éléments plus ou moins empiriques, les ravages et les dérives du «nouveau» management. Notons, d'ailleurs, que l'auteure nous invite à considérer avec méfiance ces derniers ouvrages, et particulièrement ceux d'inspirations foucaldiennes, puisqu'ils manquent bien souvent de bases empiriques solides et, finalement, considèrent aussi que ces mêmes dérives sont universelles.

Le présent ouvrage d'Evelyne Léonard vient en contrepoint à ces musiques trop connues, entendues, voire attendues par un auditoire souvent venu chercher des solutions faciles et directement applicables. Pour l'auteure, la gestion des ressources humaines est avant tout une fonction normative et pratique. La GRH énonce des «préceptes opératoires» qui vont spécifier, pour tout un chacun au sein de l'organisation, des manières de faire au regard des objectifs définis par l'organisation et qui vont réguler les relations d'emploi. La fonction GRH définit un "comment faire» au sein de l'organisation, même si ce n'est pas sa visée explicite, et, par conséquent, contribue à l'émergence d'un ordre social localisé. C'est indubitable. Mais pour autant cette fonction peut-elle se passer de réflexion? 
Peut-on naturellement imaginer que la GRH puisse s'apprendre comme l'on enseigne une technique? Peut-on définir a priori ce que doit être (ou ne pas être) la gestion des ressources humaines et ce que doit faire (ou ne pas faire) le gestionnaire? Le cas échéant, l'auteure nous rappelle à bon escient qu'en tant qu'enseignants, nous ferions du double normatif, puisque nous définirions nous-mêmes les conditions d'efficacité de ces normes. Or, nous sommes peut-être les moins bien placés pour définir ces conditions, puisque la performance est un concept dont la définition varie d'une entreprise à l'autre. De plus, si on lit entre les lignes, l'on comprendra rapidement que l'auteure considère que ce n'est pas aux professeurs d'université de répondre à ces attentes de performance.

Partant de ces constats, plutôt que de nous amener à définir ce que la GRH doit faire ou doit être, l'ouvrage nous invite à nous interroger sur ce qu'elle est, sur ce qu'elle fait, sur son rôle et sur les conditions de sa pratique. Au fil des pages, c'est toute une réflexion d'ordre "ontologique» qui nous est livrée. Mobilisant une approche analytique, l'auteure nous invite à cadrer cette fonction RH et ses activités, à identifier et à comprendre ce qui est à l'œuvre socialement et politiquement lorsqu'elle se met en œuvre dans les organisations. L'on ne s'étonnera donc pas de voir l'auteure s'éloigner des conceptions réductrices de la psychologie et du comportement organisationnel qui ont généralement cours dans les manuels prescriptifs traditionnels, et de les troquer pour un vocable ancré dans les théories des organisations et de la sociologie, sans pour autant être obscur et difficilement accessible pour l'étudiant. En effet, usant de nombreux exemples, de mises en situation plausibles, l'auteure fait émerger les concepts abstraits avec élégance, et ces derniers s'imposent à nous comme une évidence.

L'ouvrage est divisé en trois grandes parties logiquement articulées et enchâs- sées. Dans un premier temps, Evelyne Léonard définit la $\mathrm{GRH}$, tant sur le plan fonctionnel que sur le plan opérationnel. La GRH est une fonction de régulation qui participe à l'émergence d'un ordre social et à sa répétition, maintenant au travers de toutes ses pratiques, une division entre ceux qui gèrent et ceux qui sont gérés. Elle use pour se faire de moyens précis: des politiques, des pratiques, mais aussi d'une rhétorique. Ces concepts et leurs articulations sont étudiés en détail. Au passage, l'auteure rappelle que la rhétorique vise bien souvent à légitimer le management et son action et, dans bien des cas, à tenter d'occulter l'écart entre une GRH désirée et une $\mathrm{GRH}$ réelle.

Le deuxième chapitre répond en détail aux trois questions suivantes: "Quelles sont, dans et hors de I'organisation, les autres sources de normes influant sur le personnel, et comment la GRH s'inscrit-elle dans cet ensemble? Quelle est la marge de manœuvre dont disposent les gestionnaires dans ce cadre? Quelle est leur capacité à agir effectivement sur les ressources humaines et, de là, sur l'ordre social interne? ». La gestion des ressources humaines n'est évidemment pas affranchie de toute contrainte. D'une part, l'environnement organisationnel et institutionnel définit lui-même un nombre de règles $d u$ jeu auxquelles la GRH doit se conformer dans une certaine mesure. D'autre part, les membres d'une organisation ne sont pas «passifs » et réagissent naturellement aux politiques et pratiques de GRH auxquelles ils sont soumis. La GRH est donc aussi le résultat d'un rapport de forces internes et externes à l'organisation, et l'ordre auquel elle contribue est négocié, ce qui, finalement, rend ses résultats incertains, bien que réels, et ne rend pas le gestionnaire des ressources humaines seul maître du jeu.

Le troisième chapitre est peut-être le moins abouti de l'ouvrage. Toutefois, il soulève implicitement la question du devenir de la fonction. Si la GRH est produc- 
trice d'un ordre local, sa place et son rang constituent également un enjeu dans un environnement organisationnel et institutionnel en pleine transformation. La complexité croissante de certaines organisations, en particulier les multinationales, rend difficile la localisation du pouvoir de décision et la maîtrise même des règles du jeu est bien souvent l'objet de jeux politiques complexes entre acteurs soumis à des contraintes locales différentes. II s'en suit que le gestionnaire des ressources humaines est bien souvent en peine de déterminer quel est réellement son pouvoir d'action, compte tenu de I'accumulation des contraintes et des normes auxquels il doit se conformer. Ceci l'amène peut-être, comme le suggère l'auteure, à succomber aux sirènes des cabinets-conseils, et aux modes managériales dont les rhétoriques masquent toutes les contradictions et les incohérences auxquelles il doit faire face. Le gestionnaire des ressources humaines est, en effet, bien souvent amené à vivre une certaine expérience de l'impossible.

À la lecture de cet ouvrage, nous pouvons rassurer son auteure. Ce livre n'est pas « un livre de plus» à mettre sur des tablettes trop encombrées. II nous apparaît comme un incontournable qui devrait se tenir dans les mains de tout-e étudiant-e à I'université souhaitant embrasser une carrière de gestionnaire des ressources humaines. Selon nous, il permet d'entrevoir de manière fondamentale les enjeux de la fonction. Certes, d'aucuns diront qu'il ne propose pas la moindre piste d'action concrète pour le gestionnaire et que, dès lors, son utilité est toute relative. Nous leur répondrons que c'est sans doute la plus grande qualité de ce livre que de ne pas tomber dans le piège du «conseil» et de la «prescription» de l'expert. L'ouvrage ne vise qu'un seul objectif: faire état de la réalité de la fonction. Considérant sa complexité et les enjeux humains considérables auxquels elle fait face aujourd'hui, à nos yeux, il s'agit là sans conteste d'une voie pédagogique prometteuse. Si de l'ontologique, nous ne pouvons déduire le prescriptif, à tout le moins, nous pouvons espérer en déduire I'envisageable. Et, ce n'est pas rien.

\section{Sylvain Luc}

Professeur

Département des relations industrielles

Université Laval

\section{L'intervention en santé et en sécurité du travail. Pour agir en prévention dans les milieux de travail}

Sous la direction de Sylvie Montreuil, Pierre-Sébastien Fournier et Geneviève BarilGingras (2013) Québec: PUL, 530 pages.

ISBN : 978-2-7637-1878-1.

Cet ouvrage collectif, qui regroupe des spécialistes de la recherche en santé et sécurité au travail, et en intervention, examine les conditions dans lesquelles s'exerce la prévention des risques d'accidents du travail. Dans ce livre, les auteurs font un état des lieux de la situation afin de comprendre les déterminants structurels, sociaux et politiques.

Bien qu'il y ait eu des améliorations des conditions et des environnements de travail, les problématiques reliées à la santé et à la sécurité au travail n'ont pas, pour autant, diminuées. L'apparition de nouvelles maladies, telles que les maladies mentales, a amené de profondes transformations, ainsi qu'une pression accrue sur le système de santé. Les problèmes de santé ont pris une forme particulière et l'une des conséquences provient du fait que les coûts et les effets négatifs du travail sur la santé ne sont pas pris en charge par l'entreprise, mais externalisés aux travailleurs et à l'ensemble de la société.

C'est à travers un modèle d'analyse inspiré de l'ergonomie, et qui sert de fil conducteur à l'ouvrage, que les auteurs analysent la situation du travail et de ses effets sur la santé. Cette approche permet de mieux saisir les conditions dans lesquelles s'exerce la prévention des risques à la santé. 